\title{
On isometries of twin buildings
}

\section{Sebastian Bischof ${ }^{1} \cdot$ Anton Chosson $^{2}$ - Bernhard Mühlherr ${ }^{1}$}

Received: 22 November 2019 / Accepted: 3 August 2020 / Published online: 24 December 2020

() The Author(s) 2020

\begin{abstract}
A twin building consists of two buildings that are twinned by a codistance function. We prove that the local structure of a twin building uniquely determines the two buildings up to isomorphism. This has been known for twin buildings satisfying a technical condition (co).
\end{abstract}

Keywords Twin buildings $\cdot$ Local structure $\cdot$ Isometries $\cdot$ Classification

Mathematics Subject Classification 20E42 - 51E24

\section{Introduction}

Twin buildings were introduced by Ronan and Tits in the late 1980s. Their definition was motivated by the theory of Kac-Moody groups over fields. Each such group acts naturally on a pair of buildings and the action preserves an opposition relation between the chambers of the two buildings. This opposition relation shares many important properties with the opposition relation on the chambers of a spherical building. Thus, twin buildings appear to be natural generalisations of spherical buildings with infinite Weyl groups.

One of the most celebrated results in the theory of abstract buildings is Tits' classification of irreducible spherical buildings of rank at least 3 in Tits (1986). The decisive step in this classification is the proof of a local-to-global result for spherical buildings. In his survey paper Tits (1992) Tits proves several results that are inspired by his

\footnotetext{
Bernhard Mühlherr

bernhard.m.muehlherr@math.uni-giessen.de

Sebastian Bischof

sebastian.bischof@math.uni-giessen.de

Anton Chosson

anton.chosson@u-psud.fr

1 Mathematisches Institut, Arndtstraße 2, 35392 Gießen, Germany

2 IUT d'Orsay Université Paris-Sud, 91405 Orsay Cedex, France
} 
strategy in the spherical case and he discusses several obstacles for obtaining a similar local-to-global result for twin buildings. A first observation in this discussion is that the local-to-global principle seems to be valid only for 2-spherical twin buildings. But even in this case the question about the validity of the local-to-global principle remained open. Based on Tits' contributions in Tits (1992) the local-to-global principle was proved in Mühlherr and Ronan (1995) for 2-spherical twin buildings that satisfy an additional assumption, called Condition (co). In Mühlherr and Ronan (1995) Condition (co) is discussed in some detail and it turns out that it is rather mild. On the other hand, it follows from that discussion that there are affine twin buildings of type $\widetilde{C}_{2}$ that do not satisfy Condition (co).

The question whether the local-to-global principle for 2-spherical buildings holds without Condition (co) is still open at present. The main result of this paper is a contribution to the local-to-global principle without assuming any additional condition. It was proved independently by A.C. in [Ch00] and B.M. in [Mu97] but never published. In the present article we follow the basic strategy of these references. However, several contributions to the theory of twin buildings that have been made in the meantime provided various improvements of the arguments and exposition. Our motivation to publish the paper at this point is provided by the fact that it can be used to prove the local-to-global principle for 2-spherical twin buildings under a weaker assumption than Condition (co). This yields in particular the local-to-global principle for all affine twin buildings of rank at least 3 and in particular for those which do not satisfy Condition (co). This will be published in a subsequent paper. Thus, the present paper should be seen as the first in a series of two papers in which we intend to improve the main result of Mühlherr and Ronan (1995).

\section{The main result}

In order to give the precise statement of the main result it is convenient to fix some notation.

Let $(W, S)$ be a Coxeter system. We call $(W, S) 2$-spherical if $s t$ has finite order for all $s, t \in S$.

A building of type $(W, S)$ is a pair $\Delta=(\mathcal{C}, \delta)$ consisting of a non-empty set $\mathcal{C}$ and a mapping $\delta: \mathcal{C} \times \mathcal{C} \longrightarrow W$ (see Sect. 2 for the precise definition). The elements of $\mathcal{C}$ are called the chambers of $\Delta$ and the mapping $\delta$ is called the Weyl-distance. For $J \subseteq S$ and $c \in \mathcal{C}$, the set $R_{J}(c):=\{d \in \mathcal{C} \mid \delta(c, d) \in\langle J\rangle\}$ is called the $J$-residue of $c$ and for $s \in S$ the set $\mathcal{P}_{S}(c):=R_{\{s\}}(c)$ is called the $s$-panel of $c$. The set

$$
E_{2}(c):=\bigcup_{J \subseteq S,|J| \leq 2} R_{J}(c)
$$

is called the foundation of $\Delta$ at $c$. The building $\Delta$ is said to be thick if $\left|\mathcal{P}_{S}(c)\right| \geq 3$ for all $(s, c) \in S \times \mathcal{C}$.

A twin building of type $(W, S)$ is a triple $\Delta=\left(\Delta_{+}, \Delta_{-}, \delta_{*}\right)$ consisting of two buildings $\Delta_{+}=\left(\mathcal{C}_{+}, \delta_{+}\right)$and $\Delta_{-}=\left(\mathcal{C}_{-}, \delta_{-}\right)$of type $(W, S)$ and a codistance function (or twinning) 


$$
\delta_{*}:\left(\mathcal{C}_{+} \times \mathcal{C}_{-}\right) \cup\left(\mathcal{C}_{-} \times \mathcal{C}_{+}\right) \longrightarrow W
$$

and we refer to Sect. 3 for the precise definition. For a chamber $c \in \mathcal{C}_{+}$(resp. $c \in \mathcal{C}_{-}$) the set $E_{2}(c)$ denotes its foundation of $\Delta_{+}$(resp. $\Delta_{-}$) and $\Delta$ is thick if $\Delta_{+}$and $\Delta_{-}$are thick. Two chambers $c_{+} \in \mathcal{C}_{+}$and $c_{-} \in \mathcal{C}_{-}$are said to be opposite in $\Delta$ if $\delta_{*}\left(c_{+}, c_{-}\right)=1_{W}$.

Let $\Delta=\left(\left(\mathcal{C}_{+}, \delta_{+}\right),\left(\mathcal{C}_{-}, \delta_{-}\right), \delta_{*}\right)$ and $\Delta^{\prime}=\left(\left(\mathcal{C}_{+}^{\prime}, \delta_{+}^{\prime}\right),\left(\mathcal{C}_{-}^{\prime}, \delta_{-}^{\prime}\right), \delta_{*}^{\prime}\right)$ be twin buildings of type $(W, S)$ and let $\mathcal{X} \subseteq \mathcal{C}_{+} \cup \mathcal{C}_{-}, \mathcal{X}^{\prime} \subseteq \mathcal{C}_{+}^{\prime} \cup \mathcal{C}_{-}^{\prime}$ be sets of chambers of $\Delta$ and $\Delta^{\prime}$. An isometry from $\mathcal{X}$ to $\mathcal{X}^{\prime}$ is a bijection from $\mathcal{X}$ onto $\mathcal{X}^{\prime}$ which preserves signs and the Weyl-distance (resp. codistance) for each pair $(x, y) \in \mathcal{X}^{2}$.

We are now in the position to give the precise statement of our main result.

Main result: Let $(W, S)$ be a 2-spherical Coxeter system and let $\Delta=\left(\left(\mathcal{C}_{+}, \delta_{+}\right),\left(\mathcal{C}_{-}, \delta_{-}\right), \delta_{*}\right)$ and $\Delta^{\prime}=\left(\left(\mathcal{C}_{+}^{\prime}, \delta_{+}^{\prime}\right),\left(\mathcal{C}_{-}^{\prime}, \delta_{-}^{\prime}\right), \delta_{*}^{\prime}\right)$ be thick twin buildings of type $(W, S)$. Let $c_{+} \in \mathcal{C}_{+}, c_{-} \in \mathcal{C}_{-}$be opposite chambers in $\Delta$ and let $c_{+}^{\prime} \in \mathcal{C}_{+}^{\prime}, c_{-}^{\prime} \in \mathcal{C}_{-}^{\prime}$ be opposite chambers in $\Delta^{\prime}$.

Then each isometry

$$
\varphi: E_{2}\left(c_{+}\right) \cup\left\{c_{-}\right\} \rightarrow E_{2}\left(c_{+}^{\prime}\right) \cup\left\{c_{-}^{\prime}\right\}
$$

extends to an isometry

$$
\psi: \mathcal{C}_{+} \cup E_{2}\left(c_{-}\right) \rightarrow \mathcal{C}_{+}^{\prime} \cup E_{2}\left(c_{-}^{\prime}\right)
$$

Several remarks on the main result of this paper are in order.

1. Note that our main result does not assert the uniqueness of the extension $\psi$. At present, the uniqueness of $\psi$ is an open question that is most relevant for a possible proof of the local-to-global principle. Indeed, the key observation in Mühlherr and Ronan (1995) was that the extension $\psi$ is unique if $\Delta$ satisfies Condition (co).

2. A slightly weaker version of our main result was stated by Tits in the early 1990s (as Théorème 1 in Tits (1989-1990) and as Theorem 2 in Tits (1992)) and an outline of a proof is given in both references. However, as pointed out in Paragraph 2.8 of Tits (1997-1998), one of the claims made in the outline remains unclear. That our main result holds for twin buildings satisfying Condition (co) was verified by Ronan (see Theorem (7.5) in Ronan (2000)).

3. The proof of the main result combines an idea of Tits given in the outline mentioned in the previous remark with a technique that he used in Tits (1986). More concretely, for a chamber $c$ and an apartment containing $c$ in a twin building one can define two retraction mappings. We call them $\pi$ - and $\omega$-retractions. The outline in Tits (1989-1990) and Tits (1992) uses $\pi$-retractions and $\omega$-retractions are used in Tits (1986) for the proof of the local-to-global principle for spherical buildings. The key observation in this paper is that the main result can proved by using them both. 


\section{Preliminaries}

\section{Coxeter system}

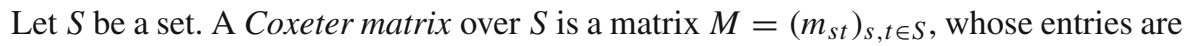
in $\mathbb{N} \cup\{\infty\}$ such that $m_{s s}=1$ for all $s \in S$ and $m_{s t}=m_{t s} \geq 2$ for all $s \neq t \in S$. For $J \subseteq S$ we set $M_{J}:=\left(m_{s t}\right)_{s, t \in J}$. The Coxeter diagram corresponding to $M$ is the labeled graph $(S, E(S))$, where $E(S)=\left\{\{s, t\} \mid m_{s t}>2\right\}$ and where each edge $\{s, t\}$ is labeled by $m_{s t}$ for all $s, t \in S$. As the Coxeter matrix and the corresponding Coxeter diagram carry the same information we do not distinguish between them formally. We call the Coxeter diagram irreducible, if the underlying graph is connected, and we call it 2-spherical, if $m_{s t}<\infty$ for all $s, t \in S$. The rank of a Coxeter diagram is the cardinality of the set of its vertices.

Let $M=\left(m_{s t}\right)_{s, t \in S}$ be a Coxeter matrix over a set $S$. A Coxeter system of type $M$ is a pair $(W, S)$ consisting of a group $W$ and a set $S \subseteq W$ of generators of $W$ such that the set $S$ and the relations $(s t)^{m_{s t}}$ for all $s, t \in S$ constitute a presentation of $W$.

Let $(W, S)$ be a Coxeter system of type $M$. The pair $(\langle J\rangle, J)$ is a Coxeter system of type $M_{J}$ (cf. (Bourbaki 1968, Ch. IV, $\S 1$ Théorème 2). For an element $w \in W$ we put $\ell(w):=\min \left\{k \in \mathbb{N}_{0} \mid \exists s_{1}, \ldots, s_{k} \in S: w=s_{1} \cdots s_{k}\right\}$. The number $\ell(w)$ is called the length of $w$. We call $J \subseteq S$ spherical if $\langle J\rangle$ is finite. Given a spherical subset $J$ of $S$, there exists a unique element of maximal length in $\langle J\rangle$, which we denote by $r_{J}$ (cf. (Abramenko and Brown 2008, Corollary 2.19)); moreover, $r_{J}$ is an involution.

Convention 2.1 For the rest of this paper let $S$ be a set, let $M$ be a Coxeter matrix over $S$ and let $(W, S)$ be a Coxeter system of type $M$.

\section{Buildings}

A building of type $(W, S)$ is a pair $\Delta=(\mathcal{C}, \delta)$ where $\mathcal{C}$ is a non-empty set and where $\delta: \mathcal{C} \times \mathcal{C} \rightarrow W$ is a distance function satisfying the following axioms, where $x, y \in \mathcal{C}$ and $w=\delta(x, y)$ :

(Bu1) $w=1_{W}$ if and only if $x=y$;

(Bu2) if $z \in \mathcal{C}$ satisfies $s:=\delta(y, z) \in S$, then $\delta(x, z) \in\{w, w s\}$, and if, furthermore, $\ell(w s)=\ell(w)+1$, then $\delta(x, z)=w s$;

(Bu3) if $s \in S$, there exists $z \in \mathcal{C}$ such that $\delta(y, z)=s$ and $\delta(x, z)=w s$.

Let $\Delta=(\mathcal{C}, \delta)$ be a building of type $(W, S)$. The rank of $\Delta$ is the rank of the underlying Coxeter system. The elements of $\mathcal{C}$ are called chambers. Given $s \in S$ and $x, y \in \mathcal{C}$, then $x$ is called $s$-adjacent to $y$, if $\delta(x, y) \in\langle s\rangle$. The chambers $x, y$ are called adjacent, if they are $s$-adjacent for some $s \in S$. A gallery joining $x$ and $y$ is a sequence $\left(x=x_{0}, \ldots, x_{k}=y\right)$ such that $x_{l-1}$ and $x_{l}$ are adjacent for any $1 \leq l \leq k$; the number $k$ is called the length of the gallery.

Given a subset $J \subseteq S$ and $x \in \mathcal{C}$, the $J$-residue of $x$ is the set $R_{J}(x):=\{y \in$ $\mathcal{C} \mid \delta(x, y) \in\langle J\rangle$. Each $J$-residue is a building of type $(\langle J\rangle, J)$ with the distance function induced by $\delta$ (cf. (Abramenko and Brown 2008, Corollary 5.30)). A residue is a subset $R$ of $\mathcal{C}$ such that there exists $J \subseteq S$ and $x \in \mathcal{C}$ with $R=R_{J}(x)$. Since the 
subset $J$ is uniquely determined by $R$, the set $J$ is called the type of $R$ and the rank of $R$ is defined to be the cardinality of $J$. A residue is called spherical if its type is a spherical subset of $S$. A panel is a residue of rank 1. An $s$-panel is a panel of type $\{s\}$ for $s \in S$. The building $\Delta$ is called thick, if each panel of $\Delta$ contains at least three chambers.

Given $x \in \mathcal{C}$ and $k \in \mathbb{N}_{0}$ then $E_{k}(x)$ denotes the union of all residues of rank at most $k$ containing $x$. It is a fact, that the set $E_{k}(x)$ determines the chamber $x$ uniquely, if $k<|S|$.

Given $x \in \mathcal{C}$ and a $J$-residue $R \subseteq \mathcal{C}$, then there exists a unique chamber $z \in R$ such that $\ell(\delta(x, y))=\ell(\delta(x, z))+\ell(\delta(z, y))$ for any $y \in R$ (cf. (Abramenko and Brown 2008, Proposition 5.34)). The chamber $z$ is called the projection of $x$ onto $R$ and is denoted by $\operatorname{proj}_{R} x$. Moreover, if $z=\operatorname{proj}_{R} x$ we have $\delta(x, y)=\delta(x, z) \delta(z, y)$ for each $y \in R$.

A subset $\Sigma \subseteq \mathcal{C}$ is called convex if $\operatorname{proj}_{P} c \in \Sigma$ for every $c \in \Sigma$ and every panel $P \subseteq \mathcal{C}$ which meets $\Sigma$. A subset $\Sigma \subseteq \mathcal{C}$ is called thin if $P \cap \Sigma$ contains exactly two chambers for every panel $P \subseteq \mathcal{C}$ which meets $\Sigma$. An apartment is a non-empty subset $\Sigma \subseteq \mathcal{C}$, which is convex and thin. It is a basic fact that in an apartment the map $\sigma_{c}: \Sigma \rightarrow W, x \mapsto \delta(c, x)$ is a bijection for any $c \in \mathcal{C}$.

\section{Chamber systems}

Let $I$ be a set. A chamber system over $I$ is a pair $\mathbf{C}=\left(\mathcal{C},\left(\sim_{i}\right)_{i \in I}\right)$ where $\mathcal{C}$ is a non-empty set whose elements are called chambers and where $\sim_{i}$ is an equivalence relation on the set of chambers for each $i \in I$. Given $i \in I$ and $c, d \in \mathcal{C}$, then $c$ is called $i$-adjacent to $d$ if $c \sim_{i} d$. The chambers $c, d$ are called adjacent if they are $i$-adjacent for some $i \in I$.

A gallery in $\mathbf{C}$ is a sequence $\left(c_{0}, \ldots, c_{k}\right)$ such that $c_{\mu} \in \mathcal{C}$ for all $0 \leq \mu \leq k$ and such that $c_{\mu-1}$ is adjacent to $c_{\mu}$ for all $1 \leq \mu \leq k$. The number $k$ is called the length of the gallery. Given a gallery $G=\left(c_{0}, \ldots, c_{k}\right)$, then we put $\beta(G):=c_{0}$ and $\varepsilon(G):=c_{k}$. If $G$ is a gallery and if $c, d \in \mathcal{C}$ such that $c=\beta(G), d=\varepsilon(G)$, then we say that $G$ is a gallery from $c$ to $d$ or $G$ joins $c$ and $d$. The chamber system $\mathbf{C}$ is said to be connected, if for any two chambers there exists a gallery joining them. A gallery $G$ will be called closed if $\beta(G)=\varepsilon(G)$.

Given a gallery $G=\left(c_{0}, \ldots, c_{k}\right)$ then $G^{-1}$ denotes the gallery $\left(c_{k}, \ldots, c_{0}\right)$ and if $H=\left(c_{0}^{\prime}, \ldots, c_{l}^{\prime}\right)$ is a gallery such that $\varepsilon(G)=\beta(H)$, then $G H$ denotes the gallery $\left(c_{0}, \ldots, c_{k}=c_{0}^{\prime}, \ldots, c_{l}^{\prime}\right)$.

Let $J$ be a subset of $I$. A $J$-gallery is a gallery $\left(c_{0}, \ldots, c_{k}\right)$ such that for each $1 \leq \mu \leq k$ there exists an index $j \in J$ with $c_{\mu-1} \sim_{j} c_{\mu}$. Given two chambers $c, d$, then we say that $c$ is $J$-equivalent with $d$ if there exists a $J$-gallery joining $c$ and $d$ and we write $c \sim{ }_{J} d$ in this case. Given a chamber $c$ and a subset $J$ of $I$ then the set $R_{J}(c):=\left\{d \in \mathcal{C} \mid c \sim{ }_{J} d\right\}$ is called the $J$-residue of $c$.

Let $\Delta=(\mathcal{C}, \delta)$ be a building of type $(W, S)$. Then we define the chamber system $\mathbf{C}(\Delta)$ as follows: The set of chambers is identified with $\mathcal{C}$ and two chambers $x, y$ are defined to be $s$-adjacent if $\delta(x, y) \in\langle s\rangle$. 


\section{Homotopy of galleries and simple connectedness}

In the context of chamber systems there is the notation of $m$-homotopy and $m$-simple connectedness for each $m \in \mathbb{N}$. In this paper we are only concerned with the case $m=2$. Therefore our definitions are always to be understood as a specialisation of the general theory to the case $m=2$.

Let $\mathbf{C}=\left(\mathcal{C},\left(\sim_{i}\right)_{i \in I}\right)$ be a chamber system over a set $I$. Two galleries $G$ and $H$ are said to be elementary homotopic if there exists two galleries $X, Y$ and two $J$-galleries $G_{0}, H_{0}$ for some $J \subseteq I$ of cardinality at most 2 such that $G=X G_{0} Y, H=X H_{0} Y$. Two galleries $G, H$ are said to be homotopic if there exists a finite sequence $G_{0}, \ldots, G_{l}$ of galleries such that $G_{0}=G, G_{l}=H$ and such that $G_{\mu-1}$ is elementary homotopic to $G_{\mu}$ for all $1 \leq \mu \leq l$.

If two galleries $G, H$ are homotopic, then it follows by definition that $\beta(G)=\beta(H)$ and $\varepsilon(G)=\varepsilon(H)$. A closed gallery $G$ is said to be null-homotopic if it is homotopic to the gallery $(\beta(G))$. The chamber system $\left(\mathcal{C},\left(\sim_{i}\right)_{i \in I}\right)$ is called simply connected if it is connected and if each closed gallery is null-homotopic.

Let $\mathcal{X} \subseteq \mathcal{C}$ and let $\mathbf{X}=\left(\mathcal{X},\left(\sim_{i}\right)_{i \in I}\right)$ be the chamber system obtained by restricting the equivalence relations $\sim_{i}$ to $\mathcal{X}$. The subset $\mathcal{X}$ will be called simply connected if the chamber system $\mathbf{X}$ is simply connected.

Proposition 2.2 Let $\Delta$ be a building of type $(W, S)$. Then the chamber system $\boldsymbol{C}(\Delta)$ is simply connected.

Proof This is (Ronan 2009, (4.3) Theorem).

\section{Twin buildings}

\section{Definitions and notations}

Let $\Delta_{+}=\left(\mathcal{C}_{+}, \delta_{+}\right), \Delta_{-}=\left(\mathcal{C}_{-}, \delta_{-}\right)$be two buildings of the same type $(W, S)$. A codistance (or a twinning) between $\Delta_{+}$and $\Delta_{-}$is a mapping $\delta_{*}:\left(\mathcal{C}_{+} \times \mathcal{C}_{-}\right) \cup$ $\left(\mathcal{C}_{-} \times \mathcal{C}_{+}\right) \rightarrow W$ satisfying the following axioms, where $\varepsilon \in\{+,-\}, x \in \mathcal{C}_{\varepsilon}, y \in \mathcal{C}_{-\varepsilon}$ and $w=\delta_{*}(x, y)$ :

(Tw1) $\delta_{*}(y, x)=w^{-1}$;

(Tw2) if $z \in \mathcal{C}_{-\varepsilon}$ is such that $s:=\delta_{-\varepsilon}(y, z) \in S$ and $\ell(w s)=\ell(w)-1$, then $\delta_{*}(x, z)=w s$

(Tw3) if $s \in S$, there exists $z \in \mathcal{C}_{-\varepsilon}$ such that $\delta_{-\varepsilon}(y, z)=s$ and $\delta_{*}(x, z)=w s$.

A twin building of type $(W, S)$ is a triple $\Delta=\left(\Delta_{+}, \Delta_{-}, \delta_{*}\right)$ where $\Delta_{+}, \Delta_{-}$are buildings of type $(W, S)$ and where $\delta_{*}$ is a twinning between $\Delta_{+}$and $\Delta_{-}$.

Convention 3.1 For the rest of this paper let $\Delta=\left(\Delta_{+}, \Delta_{-}, \delta_{*}\right)$ be a twin building of type $(W, S)$ where $\Delta_{+}=\left(\mathcal{C}_{+}, \delta_{+}\right)$and $\Delta_{-}=\left(\mathcal{C}_{-}, \delta_{-}\right)$.

We put $\mathcal{C}:=\mathcal{C}_{+} \cup \mathcal{C}_{-}$and define the distance function $\delta: \mathcal{C} \times \mathcal{C} \rightarrow W$ by setting $\delta(x, y):=\delta_{+}(x, y)\left(\operatorname{resp} . \delta_{-}(x, y), \delta_{*}(x, y)\right)$ if $x, y \in \mathcal{C}_{+}\left(\operatorname{resp} . x, y \in \mathcal{C}_{-},(x, y) \in\right.$ $\mathcal{C}_{\varepsilon} \times \mathcal{C}_{-\varepsilon}$ for some $\left.\varepsilon \in\{+,-\}\right)$. 
Given $x, y \in \mathcal{C}$ then we put $\ell(x, y):=\ell(\delta(x, y))$. If $\varepsilon \in\{+,-\}$ and $x, y \in \mathcal{C}_{\varepsilon}$, then we put $\ell_{\varepsilon}(x, y):=\ell\left(\delta_{\varepsilon}(x, y)\right)$ and for $(x, y) \in \mathcal{C}_{\varepsilon} \times \mathcal{C}_{-\varepsilon}$ we put $\ell_{*}(x, y):=$ $\ell\left(\delta_{*}(x, y)\right)$.

Let $\varepsilon \in\{+,-\}$. For $x \in \mathcal{C}_{\varepsilon}$ we put $x^{o p}:=\left\{y \in \mathcal{C}_{-\varepsilon} \mid \delta_{*}(x, y)=1_{W}\right\}$. It is a direct consequence of (Tw1) that $y \in x^{o p}$ if and only if $x \in y^{o p}$ for any pair $(x, y) \in \mathcal{C}_{\varepsilon} \times \mathcal{C}_{-\varepsilon}$. If $y \in x^{o p}$ then we say that $y$ is opposite to $x$ or that $(x, y)$ is a pair of opposite chambers.

Let $\overline{\mathcal{C}}:=\left\{\left(c_{+}, c_{-}\right) \in \mathcal{C}_{+} \times \mathcal{C}_{-} \mid \delta_{*}\left(c_{+}, c_{-}\right)=1_{W}\right\}$. Then $\left(\overline{\mathcal{C}},\left(\sim_{s}\right)_{s \in S}\right)$ is a chamber system, where $\left(c_{+}, c_{-}\right) \in \overline{\mathcal{C}}$ is $s$-adjacent $(s \in S)$ to $\left(d_{+}, d_{-}\right) \in \overline{\mathcal{C}}$ if $c_{\varepsilon}$ is $s$-adjacent to $d_{\varepsilon}$ in $\mathbf{C}\left(\Delta_{\varepsilon}\right)$ for each $\varepsilon \in\{+,-\}$. We denote this chamber system by $\operatorname{Opp}(\Delta)$. For $\bar{c}:=\left(c_{+}, c_{-}\right) \in \overline{\mathcal{C}}$ we define $E_{2}(\bar{c}):=E_{2}\left(c_{+}\right) \cup E_{2}\left(c_{-}\right)$.

A residue (resp. panel) of $\Delta$ is a residue (resp. panel) of $\Delta_{+}$or $\Delta_{-}$; given a residue $R \subseteq \mathcal{C}$ then we define its type and rank as before. Two residues $R, T \subseteq \mathcal{C}$ are called opposite if they have the same type and if there exists a pair of opposite chambers $(x, y)$ such that $x \in R, y \in T$.

Let $\varepsilon \in\{+,-\}$, let $J$ be a spherical subset of $S$ and let $R$ be a $J$-residue of $\Delta_{\varepsilon}$. Given a chamber $x \in \mathcal{C}_{-\varepsilon}$ then there exists a unique chamber $z \in R$ such that $\ell_{*}(x, y)=\ell_{*}(x, z)-\ell_{\varepsilon}(z, y)$ for any chamber $y \in R$ (cf. (Abramenko and Brown 2008 , Lemma 5.149)). The chamber $z$ is called the projection of $x$ onto $R$; it will be denoted by $\operatorname{proj}_{R} x$. Moreover, if $z=\operatorname{proj}_{R} x$ we have $\delta_{*}(x, y)=\delta_{*}(x, z) \delta_{\varepsilon}(z, y)$ for each $y \in R$.

Let $\Sigma_{+} \subseteq \mathcal{C}_{+}$and $\Sigma_{-} \subseteq \mathcal{C}_{-}$be apartments of $\Delta_{+}$and $\Delta_{-}$, respectively. Then the set $\Sigma=\Sigma_{+} \cup \Sigma_{-}$is called twin apartment if $\left|x^{o p} \cap \Sigma\right|=1$ for each $x \in \Sigma$. If $(x, y)$ is a pair of opposite chambers, then there exists a unique twin apartment containing $x$ and $y$. We will denote it by $A(x, y)$ and for $\varepsilon \in\{+,-\}$ we put $A_{\varepsilon}(x, y):=A(x, y) \cap \mathcal{C}_{\varepsilon}$. It is a fact that $A(x, y)=\{z \in \mathcal{C} \mid \delta(z, x)=\delta(z, y)\}$ (cf. Proposition 5.179 in Abramenko and Brown (2008)).

Lemma 3.2 Let $\Sigma \subseteq \mathcal{C}$ be a twin apartment, let $x \in \Sigma$ and let $R$ be a spherical residue of $\Delta$ which meets $\Sigma$. Then $\operatorname{proj}_{R} x \in \Sigma$.

Proof This is (Abramenko and Brown 2008, Lemma 5.173 (6)).

\section{Pairs of opposite spherical residues}

Throughout this subsection we assume that $R \subseteq \mathcal{C}_{+}, T \subseteq \mathcal{C}_{-}$are opposite residues and that the type $J$ of $R$ and $T$ is spherical.

Lemma 3.3 For each $x \in R$ there exists $y \in T$ such that $x$ and $y$ are opposite and we have $\delta_{*}(u, v) \in\langle J\rangle$ for all $(u, v) \in R \times T$.

Proof These are immediate consequences of (Abramenko and Brown 2008, Lemma 5.139 (1)).

Lemma 3.4 Let $(x, y) \in R \times T$. Then the following are equivalent:

(i) $\operatorname{proj}_{T} x=y$; 
(ii) $\delta_{*}(x, y)=r_{J}$;

(iii) $\operatorname{proj}_{R} y=x$.

Proof Suppose $y=\operatorname{proj}_{T} x$ and let $z \in T$ be such that $\delta_{-}(y, z)=r_{J}$. Then $\ell_{*}(x, z)=$ $\ell_{*}(x, y)-\ell\left(r_{J}\right)$ and hence $\ell_{*}(x, y) \geq \ell\left(r_{J}\right)$. As $\delta_{*}(x, y) \in\langle J\rangle$ by the previous Lemma, the claim follows.

Suppose now that $\delta_{*}(x, y)=r_{J}$ and let $z:=\operatorname{proj}_{T} x$. Since $\ell_{*}(x, z) \geq \ell_{*}(x, y)=$ $\ell\left(r_{J}\right)$ and $\delta_{*}(x, z) \in\langle J\rangle$, it follows that $\delta_{*}(x, z)=r_{J}$. Now $\ell\left(r_{J}\right)=\ell_{*}(x, y)=$ $\ell_{*}(x, z)-\ell_{-}(z, y)=\ell\left(r_{J}\right)-\ell_{-}(z, y)$ which implies $z=y$.

We have shown that $(i)$ and (ii) are equivalent; the equivalence of (ii) and (iii) follows by symmetry and we are done.

Lemma 3.5 The mappings $\operatorname{proj}_{R}^{T}: T \rightarrow R, x \mapsto \operatorname{proj}_{R} x$ and $\operatorname{proj}_{T}^{R}: R \rightarrow T, x \mapsto$ $\operatorname{proj}_{T} x$ are bijections inverse to each other.

Proof This is Proposition (4.3) in Ronan (2000).

\section{A technical result}

In this paragraph we prove a technical result which will be needed in the proof of Theorem 6.5 .

Lemma 3.6 Let $c \in \mathcal{C}_{-\varepsilon}, x \in \mathcal{C}_{\varepsilon}$ be two opposite chambers and let $(x=$ $\left.d_{0}, d_{1}, \ldots, d_{k}, d_{k+1}=d\right)$ be a gallery such that $\ell_{*}\left(c, d_{i}\right)=i$ for each $0 \leq i \leq k$ and $\ell_{*}(c, d) \leq k$. Then there exist chambers $x^{\prime}, z \in \mathcal{C}_{\varepsilon}$ such that $x^{\prime} \in c^{o p}$, $\delta_{*}(c, z)=\delta(x, z)=\delta\left(x^{\prime}, z\right)$ and $\ell\left(x^{\prime}, d\right)<k+1$.

Proof We put $w:=\delta_{\varepsilon}\left(x, d_{k}\right)$ and remark that our assumption implies $w=\delta_{*}\left(c, d_{k}\right)$. Furthermore we put $s:=\delta_{\varepsilon}\left(d_{k}, d\right)$ and let $P$ denote the $s$-panel containing $d_{k}$ and $d$. By our assumptions we have $\delta_{\varepsilon}(x, d) \in\{w, w s\}$. We have two cases:

$\ell(w s)=\ell(w)-1:$ As $\delta_{*}\left(c, d_{k}\right)=w$ it follows that $d_{k}=\operatorname{proj}_{P} c$ and $\delta_{*}(c, d)=w s$. Let $x^{\prime} \in \mathcal{C}_{\varepsilon}$ be a chamber such that $\delta_{\varepsilon}\left(x^{\prime}, d\right)=w s$. Then we have $x^{\prime} \in c^{o p}$ and $\delta_{\varepsilon}\left(x^{\prime}, d_{k}\right)=w=\delta_{\varepsilon}\left(x, d_{k}\right)=\delta_{*}\left(c, d_{k}\right)$ and $\ell_{\varepsilon}\left(x^{\prime}, d\right)=k-1<k+1$. Thus the assertion follows by setting $z:=d_{k}$.

$\ell(w s)=\ell(w)+1$ : We put $z:=\operatorname{proj}_{P} c$. As $\ell_{*}(c, d) \leq k$ it follows that $z \neq d$ and $\delta_{*}(c, d)=w$. Let $x^{\prime} \in \mathcal{C}_{\varepsilon}$ be a chamber such that $\delta_{\varepsilon}\left(x^{\prime}, d\right)=w$. Then $\delta_{\varepsilon}\left(x^{\prime}, z\right)=$ $w s=\delta_{\varepsilon}(x, z)=\delta_{*}(c, z)$, and $x^{\prime} \in c^{o p}$ and the assertion follows.

Lemma 3.7 Let $\varepsilon \in\{+,-\}, c \in \mathcal{C}_{-\varepsilon}$ and let $x, y \in c^{o p}$. Then there exist $k \in \mathbb{N}$, a sequence $x=x_{0}, \ldots, x_{k}=y$ of chambers in $c^{o p}$ and a sequence $z_{1}, \ldots, z_{k}$ of chambers in $\mathcal{C}_{\varepsilon}$ such that $\delta_{*}\left(c, z_{\lambda}\right)=\delta_{\varepsilon}\left(x_{\lambda-1}, z_{\lambda}\right)=\delta_{\varepsilon}\left(x_{\lambda}, z_{\lambda}\right)$ for each $1 \leq \lambda \leq k$.

Proof Let $\left(x=d_{0}, \ldots, d_{m}=y\right)$ be a minimal gallery joining $x$ and $y$. We will prove the assertion by induction on $m:=\ell_{\varepsilon}(x, y)$. Setting $z:=x=y$ the assertion is trivial for $m=0$ and we may assume that $m>0$.

Let $k:=\max \left\{0 \leq i \leq m \mid \ell_{*}\left(c, d_{i}\right)=i\right\}$ and put $d:=d_{k+1}$. By the previous lemma there are chambers $x^{\prime}, z \in \mathcal{C}_{\varepsilon}$ such that $x^{\prime} \in c^{o p}, \delta_{\varepsilon}(x, z)=\delta_{\varepsilon}\left(x^{\prime}, z\right)=$ $\delta_{*}(c, z)$ and $\ell_{\varepsilon}\left(x^{\prime}, d\right) \leq k$. It follows $\ell_{\varepsilon}\left(x^{\prime}, y\right)<m$ and we may apply induction to $x^{\prime}$ and $y$ in order to obtain the desired sequences $x=x_{0}, x_{1}=x^{\prime}, \ldots, x_{k}=y$ and $z_{1}=z, z_{2}, \ldots, z_{k}$ of chambers. 


\section{Isometries}

Let $(W, S)$ be 2-spherical and of rank at least 3. Let $\Delta$ be thick and let $\Delta^{\prime}=$ $\left(\Delta_{+}^{\prime}, \Delta_{-}^{\prime}, \delta_{*}^{\prime}\right)$ be a thick twin building of type $(W, S)$. We define $\mathcal{C}^{\prime}, \Delta_{+}^{\prime}, \Delta_{-}^{\prime}, \delta^{\prime}, \ell^{\prime}$ as in the case of $\Delta$.

\section{Definition and basic facts about isometries}

Let $\mathcal{X} \subseteq \mathcal{C}, \mathcal{X}^{\prime} \subseteq \mathcal{C}^{\prime}$. A mapping $\varphi: \mathcal{X} \rightarrow \mathcal{X}^{\prime}$ is called isometry if the following conditions are satisfied:

Iso1 The mapping $\varphi$ is bijective.

Iso2 For $\varepsilon \in\{+,-\}$ we have $\varphi\left(\mathcal{X} \cap \mathcal{C}_{\varepsilon}\right) \subseteq \mathcal{C}_{\varepsilon}^{\prime}$.

Iso3 If $x, y \in \mathcal{X}$ then $\delta^{\prime}(\varphi(x), \varphi(y))=\delta(x, y)$.

Given $\mathcal{X} \subseteq \mathcal{C}, \mathcal{X}^{\prime} \subseteq \mathcal{C}^{\prime}$, an isometry $\varphi: \mathcal{X} \rightarrow \mathcal{X}^{\prime}$ and $\left(y, y^{\prime}\right) \in \mathcal{C} \times \mathcal{C}^{\prime}$, then the pair $\left(y, y^{\prime}\right)$ will be called $\varphi$-admissible if the mapping $y \mapsto y^{\prime}$ extends $\varphi$ to an isometry from $\mathcal{X} \cup\{y\}$ onto $\mathcal{X}^{\prime} \cup\left\{y^{\prime}\right\}$. In particular, $(x, \varphi(x))$ is $\varphi$-admissible for any $x \in \mathcal{X}$. For $x, y \in \mathcal{X}$ with $(x, y) \in \overline{\mathcal{C}}$ we define $\varphi((x, y)):=(\varphi(x), \varphi(y))$. Since the building has rank at least three it is a fact that for $\left(x, x^{\prime}\right) \in \mathcal{C} \times \mathcal{C}^{\prime}$ and $\varphi: E_{2}(x) \rightarrow E_{2}\left(x^{\prime}\right)$ an isometry, we have $\varphi(x)=x^{\prime}$.

Lemma 4.1 Let $\mathcal{S}, \mathcal{X} \subseteq \mathcal{C}, \mathcal{S}^{\prime}, \mathcal{X}^{\prime} \subseteq \mathcal{C}^{\prime}$ be such that $\mathcal{S} \cap \mathcal{X}=\emptyset$ and $\mathcal{S}^{\prime} \cap \mathcal{X}^{\prime}=\emptyset$. Let $\varphi: \mathcal{S} \rightarrow \mathcal{S}^{\prime}$ and $\psi: \mathcal{X} \rightarrow \mathcal{X}^{\prime}$ be two isometries such that $(z, \psi(z))$ is $\varphi$-admissible for any $z \in \mathcal{X}$. Then the mapping

$$
\varphi \cup \psi: \mathcal{S} \cup \mathcal{X} \rightarrow \mathcal{S}^{\prime} \cup \mathcal{X}^{\prime}, x \mapsto \begin{cases}\varphi(x) & \text { if } x \in \mathcal{S}, \\ \psi(x) & \text { if } x \in \mathcal{X}\end{cases}
$$

is an isometry.

Proof Let $\Phi:=\varphi \cup \psi$. Clearly, $\Phi$ is a bijection satisfying (Iso2). It suffices to show, that $\delta(x, y)=\delta^{\prime}(\Phi(x), \Phi(y))$ for any $x \in \mathcal{S}, y \in \mathcal{X}$. Let $x \in \mathcal{S}$ and $y \in \mathcal{X}$. Then we have $\delta^{\prime}(\Phi(x), \Phi(y))=\delta^{\prime}(\varphi(x), \psi(y))=\delta(x, y)$, because $(y, \psi(y))$ is $\varphi$-admissible. This finishes the claim.

Lemma 4.2 Let $J$ be a spherical subset of $S$, let $R \subseteq \mathcal{C}, R^{\prime} \subseteq \mathcal{C}^{\prime}$ be $J$-residues, let $\varphi: R \rightarrow R^{\prime}$ be an isometry, and let $\left(x, x^{\prime}\right)$ be a $\varphi$-admissible pair. Then $\varphi\left(\operatorname{proj}_{R} x\right)=$ $\operatorname{proj}_{R^{\prime}} x^{\prime}$.

Proof This is Lemma (4.4) of Ronan (2000).

Lemma 4.3 Let $J$ be a spherical subset of $S$, let $R_{+}, R_{-} \subseteq \mathcal{C}\left(\right.$ resp. $\left.R_{+}^{\prime}, R_{-}^{\prime} \subseteq \mathcal{C}^{\prime}\right)$ be opposite $J$-residues in $\Delta$ (resp. $\left.\Delta^{\prime}\right)$, let $\varphi: R_{+} \cup R_{-} \rightarrow R_{+}^{\prime} \cup R_{-}^{\prime}$ be an isometry and let $\varepsilon \in\{+,-\}$. Then $\varphi(x)=\operatorname{proj}_{R_{\varepsilon}^{\prime}} \varphi\left(\operatorname{proj}_{R_{-\varepsilon}} x\right)$ for each $x \in R_{\varepsilon}$.

Proof This is a consequence of the previous Lemma and Lemma 3.5. 
Lemma 4.4 Let $x \in \mathcal{C}, x^{\prime} \in \mathcal{C}^{\prime}$, let $\Sigma \subseteq \mathcal{C}$ be an apartment containing $x$ and let $\varphi, \psi: E_{2}(x) \rightarrow E_{2}\left(x^{\prime}\right)$ be two isometries which agree on $E_{1}(x)$. If they also agree on $\Sigma \cap E_{2}(x)$, then we have $\varphi=\psi$.

Proof For each subset $J$ of $S$ of cardinality 2 we denote the restriction of $\varphi$ (resp. $\psi$ ) on $R_{J}(x)$ by $\varphi_{J}$ (resp. $\left.\psi_{J}\right)$.

Let $J \subseteq S$ be of cardinality 2 and let $\Sigma$ be as in the statement. Then $\varphi_{J}$ and $\psi_{J}$ agree on $\Sigma \cap R_{J}(x)$ which is an apartment of $R_{J}(x)$. The claim follows from Theorem 4.1.1 in Tits (1986).

Lemma 4.5 Let $\varphi_{+}: \mathcal{C}_{+} \rightarrow \mathcal{C}_{+}^{\prime}$ be a map and let $\left(\varphi_{x}: E_{2}(x) \rightarrow E_{2}\left(\varphi_{+}(x)\right)\right)_{x \in \mathcal{C}_{+}}$be a family of isometries such that $\varphi_{x}$ and $\varphi_{y}$ agree on $E_{2}(x) \cap E_{2}(y)$ whenever $x, y \in \mathcal{C}_{+}$ are adjacent. Then $\varphi_{+}$is an isometry and $\varphi_{x}$ is the restriction of $\varphi_{+}$on $E_{2}(x)$ for each $x \in \mathcal{C}_{+}$.

Proof Let $x, y \in \mathcal{C}_{+}$be such that $y \in E_{2}(x)$, then we can find a gallery ( $x=$ $x_{0}, \ldots, x_{k}=y$ ) in a rank 2 residue containing $x$ and $y$. It follows that $y \in E_{2}\left(x_{\lambda}\right)$ for each $0 \leq \lambda \leq k$ and using induction one obtains $\varphi_{x}(y)=\varphi_{y}(y)=\varphi_{+}(y)$. This shows that $\varphi_{x}$ coincides with the restriction of $\varphi_{+}$on $E_{2}(x)$.

Now we will show that $\varphi_{+}$is surjective. Let $y^{\prime} \in \mathcal{C}_{+}^{\prime}$. Let $x \in \mathcal{C}_{+}$and let $x^{\prime}:=$ $\varphi_{+}(x)$. As $\varphi_{x}: E_{2}(x) \rightarrow E_{2}\left(x^{\prime}\right)$ is an isometry, it follows that $E_{2}\left(x^{\prime}\right) \subseteq \varphi_{+}\left(\mathcal{C}_{+}\right)$. By induction on the length of a minimal gallery joining $x^{\prime}$ and $y^{\prime}$ in $\mathcal{C}_{+}^{\prime}$ it follows that $y^{\prime} \in \varphi_{+}\left(\mathcal{C}_{+}^{\prime}\right)$ and hence the surjectivity of $\varphi_{+}$.

The restriction of $\varphi_{+}$on the rank 2 residues being isometries it follows that $\varphi_{+}: \mathbf{C}\left(\Delta_{+}\right) \rightarrow \mathbf{C}\left(\Delta_{+}^{\prime}\right)$ is a 2 -covering. Now the injectivity of $\varphi_{+}$follows from Proposition 2.2.

Lemma 4.6 Let $\varphi_{+}: \mathcal{C}_{+} \rightarrow \mathcal{C}_{+}^{\prime}$ be an isometry, let $\left(x, x^{\prime}\right) \in \mathcal{C}_{-} \times \mathcal{C}_{-}^{\prime}$ and suppose that $\varphi_{+}\left(x^{o p}\right) \subseteq\left(x^{\prime}\right)^{o p}$. Then $\left(x, x^{\prime}\right)$ is a $\varphi_{+}$-admissible pair.

Proof This is Lemma (7.4) in Ronan (2000).

\section{Main results on local extensions of isometries}

In this subsection we let $\bar{c}:=\left(c_{+}, c_{-}\right) \in \overline{\mathcal{C}}, \bar{c}^{\prime}:=\left(c_{+}^{\prime}, c_{-}^{\prime}\right) \in \overline{\mathcal{C}^{\prime}}$.

Proposition 4.7 Let $\varphi: E_{2}\left(c_{+}\right) \cup\left\{c_{-}\right\} \rightarrow E_{2}\left(c_{+}^{\prime}\right) \cup\left\{c_{-}^{\prime}\right\}$ be an isometry. Then $\varphi$ extends uniquely to an isometry from $E_{2}\left(c_{+}\right) \cup E_{2}\left(c_{-}\right)$onto $E_{2}\left(c_{+}^{\prime}\right) \cup E_{2}\left(c_{-}^{\prime}\right)$.

Proof For a proof see Proposition (6.2) of Ronan (2000).

Proposition 4.8 Let $\bar{d} \in \overline{\mathcal{C}}$ such that $\bar{c}$ is adjacent to $\bar{d}$ in $\operatorname{Opp}(\Delta)$ and let $\varphi: E_{2}(\bar{c}) \rightarrow$ $E_{2}\left(\bar{c}^{\prime}\right)$ be an isometry. Then there exists a unique isometry $\psi: E_{2}(\bar{d}) \rightarrow E_{2}(\varphi(\bar{d}))$ such that $\varphi$ and $\psi$ agree on the intersection of their domains.

Proof This is Proposition (6.4) of Ronan (2000).

Theorem 4.9 Let $J$ be a subset of $S$ of cardinality at most 2 and let $R_{ \pm}:=R_{J}\left(c_{ \pm}\right)$. Let $\bar{R}:=\left(R_{+} \times R_{-}\right) \cap \overline{\mathcal{C}}$ and let $\varphi: E_{2}(\bar{c}) \rightarrow E_{2}\left(\bar{c}^{\prime}\right)$ be an isometry. Then there exists $a$ unique system of isometries $\left(\varphi_{\bar{x}}: E_{2}(\bar{x}) \rightarrow E_{2}(\varphi(\bar{x}))\right)_{\bar{x} \in \bar{R}}$ such that the following is satisfied: 
(a) $\varphi_{\bar{c}}=\varphi$;

(b) If $\bar{x}, \bar{y} \in \bar{R}$ are adjacent in $\operatorname{Opp}(\Delta)$, then $\varphi_{\bar{x}}$ and $\varphi_{\bar{y}}$ agree on the intersection of their domains.

Proof This is a consequence of Proposition (6.6) and Corollary (6.7) in Ronan (2000).

\section{Using $\operatorname{Opp}(\Delta)$ to extend isometries}

Let $\bar{c} \in \overline{\mathcal{C}}, \bar{c}^{\prime} \in \overline{\mathcal{C}^{\prime}}, \varphi: E_{2}(\bar{c}) \rightarrow E_{2}\left(\bar{c}^{\prime}\right)$ be an isometry and let $\bar{G}=(\bar{c}=$ $\left.\bar{x}_{0}, \ldots, \bar{x}_{k}=\bar{d}\right)$ be a gallery in $\operatorname{Opp}(\Delta)$. Then - by Proposition 4.8 - we obtain recursively a unique chamber $\bar{d}_{\varphi, \bar{G}}^{\prime}$ and a unique isometry $\varphi_{\bar{d}, \bar{G}}: E_{2}(\bar{d}) \rightarrow E_{2}\left(\bar{d}_{\varphi, \bar{G}}^{\prime}\right)$.

Lemma 4.10 The following hold:

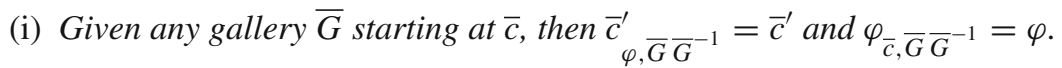

(ii) Given any closed gallery $\bar{G}$ in a rank 2 residue of $\bar{c}$, then $\bar{c}_{\varphi, \bar{G}}^{\prime}=\bar{c}^{\prime}$ and $\varphi_{\bar{c}, \bar{G}}=\varphi$.

(iii) If two galleries $\bar{G}, \bar{H}$ joining $\bar{c}$ and $\bar{d}$ are homotopic, then $\bar{d}_{\varphi, \bar{G}}^{\prime}=\bar{d}_{\varphi, \bar{H}}^{\prime}$ and $\varphi_{\bar{d}, \bar{G}}=\varphi_{\bar{d}, \bar{H}}$.

Proof Part (i) follows from the uniqueness assertion in Proposition 4.8; part (ii) follows from Theorem 4.9, and part (iii) is a consequence of (i) and (ii).

Proposition 4.11 Let $\overline{\mathcal{X}} \subseteq \overline{\mathcal{C}}$ be simply connected and suppose that $\overline{\mathcal{C}} \in \overline{\mathcal{X}}$. Then there exists a mapping $\bar{\varphi}: \overline{\mathcal{X}} \rightarrow \overline{\mathcal{C}^{\prime}}$ and a system of isometries $\left(\varphi_{\bar{x}}: E_{2}(\bar{x}) \rightarrow E_{2}(\bar{\varphi}(\bar{x}))\right)_{\bar{x} \in \overline{\mathcal{X}}}$ such that $\varphi_{\bar{c}}=\varphi$ and such that $\varphi_{\bar{x}}$ and $\varphi_{\bar{y}}$ agree on the intersection of their domains for any two adjacent chambers $\bar{x}, \bar{y} \in \overline{\mathcal{X}}$. The mapping $\bar{\varphi}$ and the family of isometries $\varphi_{\bar{x}}$ are uniquely determined by these properties.

Proof As $\overline{\mathcal{X}}$ is simply connected it is connected by definition. Given $\bar{x} \in \overline{\mathcal{X}}$ we obtain for each gallery $\bar{G}$ joining $\bar{c}$ and $\bar{x}$ a unique chamber $\bar{x}_{\varphi, \bar{G}}^{\prime}$ and an isometry $\varphi_{\bar{x}, \bar{G}}: E_{2}(\bar{x}) \rightarrow E_{2}\left(\bar{x}_{\varphi, \bar{G}}^{\prime}\right)$. It follows by part (iii) of the previous Lemma that $\bar{x}_{\bar{G}}^{\prime}=\bar{x}_{\bar{H}}^{\prime}$ for any two galleries $\bar{G}, \bar{H}$ from $\bar{c}$ to $\bar{x}$ because $\overline{\mathcal{X}}$ is simply connected. Thus we obtain a mapping $\bar{\varphi}$ and a system of isometries $(\varphi \bar{x})_{\bar{x} \in \overline{\mathcal{X}}}$.

Let $\bar{x}, \bar{y} \in \overline{\mathcal{X}}$ be adjacent. By considering a gallery joining $\bar{c}$ and $\bar{x}$ which passes through $\bar{y}$ it is seen that it follows by construction that $\varphi_{\bar{x}}$ and $\varphi_{\bar{y}}$ agree on the intersection of their domains.

The uniqueness of $\bar{\varphi}$ and $\left(\varphi_{\bar{x}}\right)_{\bar{x} \in \overline{\mathcal{X}}}$ follows from the uniqueness assertion of Proposition 4.8 and an obvious induction.

\section{Retractions}

Convention 5.1 For the rest of this paper let $(W, S)$ be 2-spherical and of rank at least 3. Furthermore, let $\Delta$ be thick and let $\Delta^{\prime}=\left(\Delta_{+}^{\prime}, \Delta_{-}^{\prime}, \delta_{*}^{\prime}\right)$ be a thick twin building of type $(W, S)$. We define $\mathcal{C}^{\prime}, \Delta_{+}^{\prime}, \Delta_{-}^{\prime}, \delta^{\prime}, \ell^{\prime}$ as in the case of $\Delta$. 


\section{$\pi$-retractions}

Let $c \in \mathcal{C}_{-}$, let $\Sigma \subseteq \mathcal{C}_{-}$be an apartment of $\Delta_{-}$containing $c$ and put $\gamma:=(c, \Sigma)$. Then we define the mapping $\pi_{\gamma}: \mathcal{C}_{+} \rightarrow \Sigma$ via $\delta_{-}\left(c, \pi_{\gamma}(x)\right)=\delta_{*}(c, x)$ for all $x \in \mathcal{C}_{+}$ and we put $\Pi_{\gamma}:=\left\{\left(x, \pi_{\gamma}(x)\right) \mid x \in \mathcal{C}_{+}\right\}$.

Lemma 5.2 Let $\gamma=(c, \Sigma)$ be as above, then the following hold:

( $\pi 1) \pi_{\gamma}$ preserves $s$-adjacency.

$(\pi 2)$ The chamber $\pi_{\gamma}(x)$ is opposite to $x$ for each chamber $x \in \mathcal{C}_{+}$.

( $\pi 3)$ Given $x \in \mathcal{C}_{+}$, then $c \in A\left(x, \pi_{\gamma}(x)\right)$.

Proof The first two assertions are proved in Lemma (7.1) in Ronan (2000). For the third assertion we notice that $\delta_{-}\left(c, \pi_{\gamma}(x)\right)=\delta_{*}(c, x)$ by definition and hence the claim follows.

Lemma 5.3 Let $\gamma=(c, \Sigma)$ be as above, then the mapping $\mathcal{C}_{+} \rightarrow \Pi_{\gamma}, x \mapsto$ $\left(x, \pi_{\gamma}(x)\right)$ is an s-adjacence preserving bijection. In particular, $\Pi_{\gamma}$ is a simply connected subset of $\overline{\mathcal{C}}$.

Proof The first statement is immediate from Lemma 5.2. The second follows from Proposition 2.2.

\section{œ-retractions}

Let $\bar{c}:=\left(c_{+}, c_{-}\right)$be a pair of opposite chambers and let $\Sigma=A_{-}\left(c_{+}, c_{-}\right)$. Then we define the mapping $\omega_{\bar{c}}: \mathcal{C}_{+} \rightarrow \Sigma$ via $\delta_{-}\left(c_{-}, \omega_{\bar{c}}(x)\right)=\delta_{+}\left(c_{+}, x\right)$ for all $x \in \mathcal{C}_{+}$. Furthermore we set $\Omega_{\bar{c}}:=\left\{\left(x, \omega_{\bar{c}}(x)\right) \mid x \in \mathcal{C}_{+}\right\}$. A gallery $\left(\bar{x}=\bar{x}_{0}, \ldots, \bar{x}_{k}=\bar{y}\right)$ in $\operatorname{Opp}(\Delta)$ will be called $\omega$-gallery if there exists a chamber $\bar{c} \in \overline{\mathcal{C}}$ such that $\bar{x}_{\lambda} \in \Omega_{\bar{c}}$ for each $0 \leq \lambda \leq k$.

Lemma 5.4 Let $\bar{c} \in \overline{\mathcal{C}}$. Then the following hold:

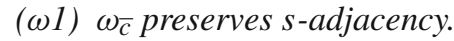

( $\omega 2)$ The chamber $\omega_{\bar{c}}(x)$ is opposite to $x$ for each chamber $x \in \mathcal{C}_{+}$.

( $\omega 3)$ Given $x \in \mathcal{C}_{+}$, then $c_{+} \in A\left(x, \omega_{\bar{c}}(x)\right)$.

Proof Let $x, y \in \mathcal{C}_{+}$and $s \in S$ such that $x, y$ are $s$-adjacent, and let $w \in W$ such that $\delta_{+}\left(c_{+}, x\right)=w$. Then $\delta_{+}\left(c_{+}, y\right) \in\{w, w s\}$ by (Bu2). If $\delta_{+}\left(c_{+}, y\right)=w$ then $\delta_{-}\left(c_{-}, \omega_{\bar{c}}(x)\right)=\delta_{-}\left(c_{-}, \omega_{\bar{c}}(y)\right)$. Since $c_{-}, \omega_{\bar{c}}(x), \omega_{\bar{c}}(y) \in \Sigma$ we obtain $\omega_{\bar{c}}(x)=$ $\omega_{\bar{c}}(y)$. Now we assume that $\delta_{+}\left(c_{+}, y\right)=w s$. Let $P$ be the $s$-panel containing $\omega_{\bar{c}}(y)$. Since $\omega_{\bar{c}}(y) \in P \cap \Sigma$ we obtain $|P \cap \Sigma|=2$ because any apartment is thin. Let $\omega_{\bar{c}}(y) \neq$ $y^{\prime} \in P \cap \Sigma$. Using (Bu2) we obtain $\delta_{-}\left(c_{-}, y^{\prime}\right) \in\{w, w s\}$. Since $c_{-}, y^{\prime}, \omega_{\bar{c}}(x) \in \Sigma$ and $\delta_{-}\left(c_{-}, y^{\prime}\right)=\delta_{-}\left(c_{-}, \omega_{\bar{c}}(x)\right)$, we obtain $y^{\prime}=\omega_{\bar{c}}(x)$ as above. Thus $\omega_{\bar{c}}$ preserves $s$-adjacency.

Let $x \in \mathcal{C}_{+}$and $w \in W$ such that $\delta_{+}\left(x, c_{+}\right)=w$. Then $\delta_{-}\left(c_{-}, \omega_{\bar{c}}(x)\right)=$ $\delta_{+}\left(c_{+}, x\right)=w^{-1}$. Since $\omega_{\bar{c}}(x) \in A\left(c_{+}, c_{-}\right)$we have $\delta_{*}\left(\omega_{\bar{c}}(x), c_{+}\right)=\delta_{-}\left(\omega_{\bar{c}}(x), c_{-}\right)=$ $w$. Now we have $\delta_{*}\left(\omega_{\bar{c}}(x), x\right)=w w^{-1}=1_{W}$ by Lemma 5.140 of Abramenko and Brown (2008) and the claim follows. 
Let $x \in \mathcal{C}_{+}$. Since $\omega_{\bar{c}} \in A\left(c_{+}, c_{-}\right)$we obtain $\delta_{*}\left(c_{+}, \omega_{\bar{c}}(x)\right)=\delta_{-}\left(c_{-}, \omega_{\bar{c}}(x)\right)$. Furthermore, we have $\delta_{+}\left(c_{+}, x\right)=\delta_{-}\left(c_{-}, \omega_{\bar{c}}(x)\right)$. Combining these two facts we obtain $c_{+} \in A\left(x, \omega_{\bar{c}}(x)\right)$ as required.

Lemma 5.5 Let $P$ be an s-panel in $\Delta_{+}$, let $x, y \in P$ be such that $\ell_{+}\left(c_{+}, y\right)=$ $\ell_{+}\left(c_{+}, x\right)+1$ and let $Q$ denote the s-panel of $\Delta_{-}$containing $\omega_{\bar{c}}(x)$ and $\omega_{\bar{c}}(y)$. Then the following hold:

(i) $\operatorname{proj}_{P} c_{+}=x$;

(ii) $\operatorname{proj}_{Q} c_{+}=\omega_{\bar{c}}(y)$;

(iii) $\operatorname{proj}_{P} \omega_{\bar{c}}(y)=x$;

(iv) $\operatorname{proj}_{Q} x=\omega_{\bar{c}}(y)$.

Proof Part $(i)$ follows from $\ell_{+}\left(c_{+}, y\right)=\ell_{+}\left(c_{+}, x\right)+1$. Since $c_{+} \in A\left(y, \omega_{\bar{c}}(y)\right) \cap$ $A\left(x, \omega_{\bar{c}}(x)\right)$ we have $\ell_{*}\left(c_{+}, \omega_{\bar{c}}(y)\right)=\ell_{+}\left(c_{+}, y\right)=\ell_{+}\left(c_{+}, x\right)+1=\ell_{*}\left(c_{+}, \omega_{\bar{c}}(x)\right)+$ 1 which yields part (ii). To prove part (iii) we use the fact that $c_{+} \in A\left(y, \omega_{\bar{c}}(y)\right)$. As $\operatorname{proj}_{P} c_{+}=x$, it follows by Lemma 3.2, that $x \in A\left(y, \omega_{\bar{c}}(y)\right)$. Applying Lemma 3.2 again we obtain that $\operatorname{proj}_{P} \omega_{\bar{c}}(y) \in\{x, y\}$, since $A_{+}\left(y, \omega_{\bar{c}}(y)\right)$ is thin. As $\ell_{*}\left(y, \omega_{\bar{c}}(y)\right)=0$ we have $\operatorname{proj}_{P} \omega_{\bar{c}}(y)=x$ as claimed. Part (iv) follows now from part (iii) and Lemma 3.5.

Lemma 5.6 The mapping $\mathcal{C}_{+} \rightarrow \Omega_{\bar{c}}: x \mapsto\left(x, \omega_{\bar{c}}(x)\right)$ is an s-adjacence preserving bijection between $\mathcal{C}_{+}$and $\Omega_{\bar{c}}$. In particular, $\Omega_{\bar{c}}$ is a simply connected subset of $\overline{\mathcal{C}}$.

Proof The first statement is immediate from Lemma 5.4. The second follows from Proposition 2.2.

Lemma 5.7 Let $c \in \mathcal{C}_{-}$, let $\Sigma$ be an apartment of $\Delta_{-}$containing $c$ and let $\gamma:=$ $(c, \Sigma)$. Let $x, y \in c^{o p}$ and suppose that there exists a chamber $z \in \mathcal{C}_{+}$such that $\delta_{+}(x, z)=\delta_{*}(c, z)=\delta_{+}(y, z)$. Then there exists an $\omega$-gallery joining $(x, c)$ and $(y, c)$ in $\left.\Pi_{\gamma} \cap \Omega_{\left(z, \pi_{\gamma}\right.}(z)\right)$.

Proof We put $\bar{z}:=\left(z, \pi_{\gamma}(z)\right)$. Then we obtain that $\omega_{\bar{z}}(z)=\pi_{\gamma}(z), \omega_{\bar{z}}(x)=\pi_{\gamma}(x)=$ $c$ and $\delta_{-}\left(\omega_{\bar{z}}(x), \omega_{\bar{z}}(z)\right)=\delta_{+}(x, z)=\delta_{-}\left(\pi_{\gamma}(x), \pi_{\gamma}(z)\right)$. Since $\pi_{\gamma}$ and $\omega_{\bar{z}}$ preserve $s$-adjacency by $(\pi 1)$ and $(\omega 1)$, it follows that they map any chamber on a minimal gallery joining $x$ any $z$ to a chamber on a minimal gallery joining $\pi_{\gamma}(x)=\omega_{\bar{z}}(x)$ to $\pi_{\gamma}(z)=\omega_{\bar{z}}(z)$. Thus we obtain $\pi_{\gamma}(v)=\omega_{\bar{z}}(v)$ for each chamber $v$ on a minimal gallery joining $x$ and $z$. The same is true for $y$ instead of $x$ and we obtain $\pi_{\gamma}(u)=\omega_{\bar{z}}(u)$ for each chamber $u$ on a minimal gallery joining $y$ and $z$. This yields the claim.

\section{Constructing an isometry}

We recall that the set $S$ has at least three elements. In this subsection let $\bar{c}:=\left(c_{+}, c_{-}\right) \in$ $\overline{\mathcal{C}}, \bar{c}^{\prime}:=\left(c_{+}^{\prime}, c_{-}^{\prime}\right) \in \overline{\mathcal{C}^{\prime}}$ and let $\varphi: E_{2}(\bar{c}) \rightarrow E_{2}\left(\bar{c}^{\prime}\right)$ be an isometry. We set $\Sigma:=$ $A_{-}\left(c_{+}, c_{-}\right), \Sigma^{\prime}:=A_{-}\left(c_{+}^{\prime}, c_{-}^{\prime}\right)$ and denote the unique isometry from $\Sigma$ onto $\Sigma^{\prime}$ extending the mapping $c_{-} \mapsto c_{-}^{\prime}$ by $\alpha$. We set $\omega:=\omega_{\bar{c}}, \omega^{\prime}:=\omega_{\bar{c}^{\prime}}$ and $\Omega:=\Omega_{\bar{c}}$. For $x \in \mathcal{C}_{+}$we put $\bar{x}:=(x, \omega(x))$.

By Lemma 5.6 and Proposition 4.11 we get a mapping $\bar{\varphi}: \Omega \rightarrow \overline{\mathcal{C}^{\prime}}$ and a system of isometries $\left(\varphi_{\bar{x}}: E_{2}(\bar{x}) \rightarrow E_{2}(\bar{\varphi}(\bar{x}))\right)_{x \in \mathcal{C}_{+}}$such that 
(i) $\varphi_{\bar{c}}=\varphi$;

(ii) $\varphi_{\bar{x}}$ and $\varphi_{\bar{y}}$ coincide on the intersection of their domains whenever $x, y$ are adjacent.

Furthermore, we define the mapping $\varphi_{+}: \mathcal{C}_{+} \rightarrow \mathcal{C}_{+}^{\prime}, x \mapsto \varphi_{\bar{x}}(x)$ and denote the restriction of $\varphi_{\bar{x}}$ on $E_{2}(x)$ by $\varphi_{x}$.

Lemma 6.1 The mapping $\varphi_{+}$is an isometry from $\mathcal{C}_{+}$to $\mathcal{C}_{+}^{\prime}$ and $\varphi_{x}$ is the restriction of $\varphi_{+}$on $E_{2}(x)$ for each $x \in \mathcal{C}_{+}$.

Proof Given two adjacent chambers $x, y \in \mathcal{C}_{+}$, then $\bar{x}$ and $\bar{y}$ are adjacent. By property (ii) above it follows that $\varphi_{x}$ and $\varphi_{y}$ coincide on $E_{2}(x) \cap E_{2}(y)$. This shows that $\varphi_{+}$ and $\left(\varphi_{x}\right)_{x \in \mathcal{C}_{+}}$satisfy the conditions of Lemma 4.5 and we are done.

Lemma 6.2 Let $P \subseteq \mathcal{C}_{+}, P^{\prime} \subseteq \mathcal{C}_{+}^{\prime}$ be panels of $\Delta_{+}$and $\Delta_{+}^{\prime}$ having the same type and let $x:=\operatorname{proj}_{P} c_{+}, x^{\prime}:=\operatorname{proj}_{P^{\prime}} c_{+}^{\prime}$. Suppose that $\delta_{+}\left(c_{+}, x\right)=\delta_{+}^{\prime}\left(c_{+}^{\prime}, x^{\prime}\right)$ and let $\psi: E_{2}((x, \omega(x))) \rightarrow E_{2}\left(\left(x^{\prime}, \omega^{\prime}\left(x^{\prime}\right)\right)\right)$ be an isometry. Given $y \in P$, then $\psi(\omega(y))=$ $\omega^{\prime}(\psi(y))$.

Proof If $x=y$ there is nothing to prove, so we may assume that $x \neq y$. As $x=$ $\operatorname{proj}_{P} c_{+}$we have $\ell_{+}\left(c_{+}, y\right)=\ell_{+}\left(c_{+}, x\right)+1$. We put $y^{\prime}:=\psi(y)$. We have $y^{\prime} \in P^{\prime}$ and $x^{\prime} \neq y^{\prime}$ because $\psi$ is an isometry. As $x^{\prime}=\operatorname{proj}_{P^{\prime}} c_{+}^{\prime}$ it follows $\ell_{+}^{\prime}\left(c_{+}^{\prime}, y^{\prime}\right)=$ $\ell_{+}^{\prime}\left(c_{+}^{\prime}, x^{\prime}\right)+1$. Let $Q$ (resp. $\left.Q^{\prime}\right)$ denote the panel containing $\omega(x)$ (resp. $\omega^{\prime}\left(x^{\prime}\right)$ ) opposite to $P$ (resp. $P^{\prime}$ ). By Lemma 5.5 and Lemma 4.3 it follows that $\psi(\omega(y))=$ $\operatorname{proj}_{Q^{\prime}} \psi\left(\operatorname{proj}_{P} \omega(y)\right)=\operatorname{proj}_{Q^{\prime}} \psi(x)=\operatorname{proj}_{Q^{\prime}} x^{\prime}=\omega^{\prime}\left(y^{\prime}\right)$ which yields the claim.

Proposition 6.3 Let $x, y \in \mathcal{C}_{+}$be such that $\omega(x)=\omega(y)$, then the restrictions of $\varphi_{\bar{x}}$ and $\varphi_{\bar{y}}$ on $E_{2}(\omega(x))$ coincide.

Proof Using Lemma 6.2 it follows by induction on $\ell_{+}\left(c_{+}, u\right)$ that $\varphi_{\bar{u}}(\omega(u))=$ $\omega^{\prime}\left(\varphi_{+}(u)\right)$ for each $u \in \mathcal{C}_{+}$. As $\varphi_{+}$is an isometry mapping $c_{+}$onto $c_{+}^{\prime}$ (cf. Lemma 6.1) it follows that $\alpha(\omega(u))=\omega^{\prime}\left(\varphi_{+}(u)\right)$ for each $u \in \mathcal{C}_{+}$.

Let $u \in \mathcal{C}_{+}$and let $z \in E_{2}(\omega(u)) \cap \Sigma$. Then there exists $v \in E_{2}(u)$ with $z=\omega(v)$. Let $\left(u=x_{0}, \ldots, x_{k}=v\right)$ be a gallery in a rank 2 residue joining $u$ and $v$. It follows that $v \in E_{2}\left(x_{\lambda}\right)$ and hence $z \in E_{2}\left(\omega\left(x_{\lambda}\right)\right)$ for each $0 \leq \lambda \leq k$. Using property (ii) of the system $\left(\varphi_{\bar{u}}\right)_{u \in \mathcal{C}_{+}}$, it follows by induction on $k$ that $\varphi_{\bar{u}}(z)=\varphi_{\bar{v}}(z)$. Combining this with the previous considerations we obtain $\varphi_{\bar{u}}(z)=\alpha(z)$ for each $z \in E_{2}(\omega(u)) \cap \Sigma$.

We complete the proof of the proposition by induction on $\ell_{+}\left(c_{+}, x\right)=\ell_{+}\left(c_{+}, y\right)$. If $\ell_{+}\left(c_{+}, x\right)=0$ then $x=c_{+}=y$ and there is nothing to prove. Let $\ell_{+}\left(c_{+}, x\right)>0$, then there exists $s \in S$ such that $\ell\left(\delta_{+}\left(c_{+}, x\right) s\right)=\ell_{+}\left(c_{+}, x\right)-1$. Let $P_{x}, P_{y}$ denote the $s$-panels containing $x$ and $y$, respectively, and put $x_{1}:=\operatorname{proj}_{P_{x}} c_{+}, y_{1}:=\operatorname{proj}_{P_{y}} c_{+}$. Then $\ell_{+}\left(c_{+}, x_{1}\right)=\ell_{+}\left(c_{+}, x\right)-1$ and we obtain $\omega\left(x_{1}\right)=\omega\left(y_{1}\right)$. Using property $($ ii $)$ of the system $\left(\varphi_{\bar{x}}\right)_{x \in \mathcal{C}_{+}}$and the induction assumption we obtain $\varphi_{\bar{x}}(z)=\varphi_{\bar{x}_{1}}(z)=$ $\varphi_{\bar{y}_{1}}(z)=\varphi_{\bar{y}}(z)$ for each $z \in E_{1}(\omega(x))$. By the previous considerations we have that $\varphi_{\bar{x}}$ and $\varphi_{\bar{y}}$ agree on $E_{2}(\omega(x)) \cap \Sigma$ and therefore the claim follows from Lemma 4.4.

A consequence of Proposition 6.3 is the following corollary which will be needed in the next subsection. 
Corollary 6.4 Let $\bar{x}:=(x, z), \bar{y}:=(y, z) \in \overline{\mathcal{C}}$, let $\bar{x}^{\prime} \in \overline{\mathcal{C}^{\prime}}$ and let $\psi: E_{2}(\bar{x}) \rightarrow E_{2}\left(\bar{x}^{\prime}\right)$ be an isometry. Let $\bar{G}$ be an $\omega$-gallery joining $\bar{x}$ and $\bar{y}$ in $\operatorname{Opp}(\Delta)$. Then $\psi_{\bar{y}, \bar{G}}$ and $\psi$ coincide on $E_{2}(z)$.

Proof Since $\omega(x)=z=\omega(y)$, the claim follows from Proposition 6.3 and the definition of $\psi_{\bar{y}, \bar{G}}$.

\section{Proof of the main theorem}

Theorem 6.5 Let $\bar{c}:=\left(c_{+}, c_{-}\right) \in \overline{\mathcal{C}}, \bar{c}^{\prime}:=\left(c_{+}^{\prime}, c_{-}^{\prime}\right) \in \overline{\mathcal{C}^{\prime}}$. Then every isometry $\varphi: E_{2}\left(c_{+}\right) \cup\left\{c_{-}\right\} \rightarrow E_{2}\left(c_{+}^{\prime}\right) \cup\left\{c_{-}^{\prime}\right\}$ extends to an isometry from $\mathcal{C}_{+} \cup E_{2}\left(c_{-}\right)$onto $\mathcal{C}_{+}^{\prime} \cup E_{2}\left(c_{-}^{\prime}\right)$.

Proof By Proposition 4.7 the isometry $\varphi$ extends to an isometry from $E_{2}(\bar{c})$ onto $E_{2}\left(\bar{c}^{\prime}\right)$. We choose an apartment $\Sigma \subseteq \mathcal{C}_{-}$containing $c_{-}$and set $\pi:=\pi_{\left(c_{-}, \Sigma\right)}, \Pi:=$ $\Pi_{\left(c_{-}, \Sigma\right)}$. For $x \in \mathcal{C}_{+}$we put $\bar{x}:=(x, \pi(x))$. By Lemma 5.3 and Proposition 4.11 we obtain a mapping $\bar{\varphi}: \Pi \rightarrow \overline{\mathcal{C}^{\prime}}$ and a system of isometries $\left(\varphi_{\bar{x}}: E_{2}(\bar{x}) \rightarrow E_{2}(\bar{\varphi}(\bar{x}))\right)_{x \in \mathcal{C}_{+}}$with the properties $(i)$ and $(i i)$ of the previous subsection. We define the mapping $\varphi_{+}: \mathcal{C}_{+} \rightarrow \mathcal{C}_{+}^{\prime}, x \mapsto \varphi_{\bar{x}}(x)$ and we denote the restriction of $\varphi_{\bar{x}}$ on $E_{2}(x)$ by $\varphi_{x}$. Then $\varphi_{+}$is an isometry from $\mathcal{C}_{+}$onto $\mathcal{C}_{+}^{\prime}$ and $\varphi_{+}, \varphi_{x}$ agree on $E_{2}(x)$ for each $x \in \mathcal{C}_{+}$by Lemma 6.1.

Let $x, y \in c_{-}^{o p}$. Using Lemma 3.7 there exist $k \in \mathbb{N}$, a sequence $x_{0}:=x, \ldots, x_{k}:=$ $y$ of chambers in $c_{-}^{o p}$ and a sequence $z_{1}, \ldots, z_{k}$ of chambers in $\mathcal{C}_{+}$such that $\delta_{*}\left(c_{-}, z_{\lambda}\right)=\delta_{+}\left(x_{\lambda-1}, z_{\lambda}\right)=\delta_{+}\left(x_{\lambda}, z_{\lambda}\right)$ for each $1 \leq \lambda \leq k$. By Lemma 5.7 there exists for any $1 \leq \lambda \leq k$ an $\omega$-gallery joining $\left(x_{\lambda-1}, c_{-}\right)$and $\left(x_{\lambda}, c_{-}\right)$in $\Pi_{\gamma} \cap \Omega_{\left(z_{\lambda}, \pi_{\gamma}\left(z_{\lambda}\right)\right)}$. Now we obtain that $\varphi_{\bar{x}}, \varphi_{\bar{y}}$ agree on $E_{2}\left(c_{-}\right)$by Corollary 6.4. We let $\varphi_{-}: E_{2}\left(c_{-}\right) \rightarrow E_{2}\left(c_{-}^{\prime}\right), z \mapsto \varphi_{\bar{x}}(z)$ denote this common restriction for some $x \in c_{-}^{o p}$ and for $z \in E_{2}\left(c_{-}\right)$we put $z^{\prime}:=\varphi_{-}(z)$.

We want to show now, that $\varphi_{+}\left(z^{o p}\right) \subseteq\left(z^{\prime}\right)^{o p}$ for each $z \in E_{2}\left(c_{-}\right)$. Let $v \in z^{o p}$ then there exists $x \in c_{-}^{o p}$ such that $v \in E_{2}(x)$ by Lemma 3.3. Since $\varphi_{+}(v)=\varphi_{x}(v), \varphi_{x}=$ $\left.\varphi_{\bar{x}}\right|_{E_{2}(x)}$ and since $\varphi_{\bar{x}}$ is an isometry from $E_{2}(\bar{x})$ onto $E_{2}(\bar{\varphi}(\bar{x}))$ whose restriction on $E_{2}\left(c_{-}\right)$is $\varphi_{-}$it follows that $\varphi_{+}(v) \in\left(z^{\prime}\right)^{o p}$.

By Lemma 4.6 the pair $\left(z, z^{\prime}\right)=\left(z, \varphi_{-}(z)\right)$ is $\varphi_{+}$-admissible. Applying Lemma 4.1 to the isometries $\varphi_{+}$and $\varphi_{-}$we obtain an isometry $\varphi_{+} \cup \varphi_{-}$as required.

Funding Open Access funding enabled and organized by Projekt DEAL.

Open Access This article is licensed under a Creative Commons Attribution 4.0 International License, which permits use, sharing, adaptation, distribution and reproduction in any medium or format, as long as you give appropriate credit to the original author(s) and the source, provide a link to the Creative Commons licence, and indicate if changes were made. The images or other third party material in this article are included in the article's Creative Commons licence, unless indicated otherwise in a credit line to the material. If material is not included in the article's Creative Commons licence and your intended use is not permitted by statutory regulation or exceeds the permitted use, you will need to obtain permission directly from the copyright holder. To view a copy of this licence, visit http://creativecommons.org/licenses/by/4.0/. 


\section{References}

Abramenko, P., Brown, K.S.: Buildings-Theory and Applications. Springer GTM 248, (2008)

Bourbaki, N.: Groupes et Algébres de Lie, Chapitres 4,5 et 6. Hermann, Paris (1968)

Chosson, A.: Isométries dans les Immeubles Jumelés et Construction d'automorphismes de groupes de Kac-Moody. Thèse de doctorat, Amiens (2000)

Mühlherr, B.: An alternative proof of the extension theorem for twin buildings. Preprint, Dortmund, 17 pages (1997)

Mühlherr, B., Ronan, M.: Local to global structure in twin buildings. Invent. Math. 122, 71-81 (1995)

Ronan, M.: Lectures on Buildings (Updated and Revised). University of Chicago Press, Chicago (2009)

Ronan, M.: Local isometries of twin buildings. Math. Z. 234, 435-455 (2000)

Tits, J.: Buildings of spherical type and finite BN-pairs. Lecture Notes in Mathematics 386, Springer Verlag 1974. $2^{\text {nd }}$ edition (1986)

Tits, J.: Immeubles jumelés (Suite). Annuaire du Collège de France, 90e, année, 1989-1990, 87-103

Tits, J.: Twin Buildings and groups of Kac-Moody type. In M. W. Liebeck and J. Saxl (eds.), Groups, Combinatorics and Geometry (Durham 1990), London Math. Soc. Lecture Notes Ser. 165, Cambridge University Press, (1992), 249-286

Tits, J.: Immeubles jumelés: théorèmes d'existence. Annuaire du Collège de France, 1997-1998, 97-112

Publisher's Note Springer Nature remains neutral with regard to jurisdictional claims in published maps and institutional affiliations. 